\title{
The Epipactis helleborine Group (Orchidaceae): An Overview of Recent Taxonomic Changes, with an Updated List of Currently Accepted Taxa
}

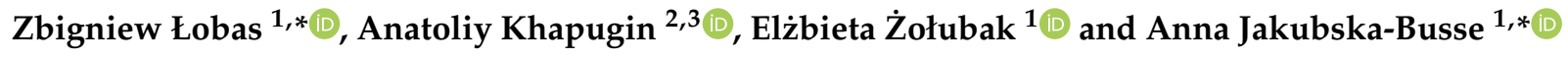 \\ 1 Department of Botany, Faculty of Biological Sciences, University of Wroclaw, 50-328 Wroclaw, Poland; \\ elzbieta.zolubak@uwr.edu.pl \\ 2 Institute of Environmental and Agricultural Biology (X-BIO), Tyumen State University, \\ 625003 Tyumen, Russia; hapugin88@yandex.ru \\ 3 Joint Directorate of the Mordovia State Nature Reserve and National Park "Smolny", 430005 Saransk, Russia \\ * Correspondence: zbigniew.lobas@uwr.edu.pl (Z.Ł.); anna.jakubska-busse@uwr.edu.pl (A.J.-B.)
}

check for

updates

Citation: Łobas, Z.; Khapugin, A.; Żołubak, E.; Jakubska-Busse, A. The Epipactis helleborine Group

(Orchidaceae): An Overview of

Recent Taxonomic Changes, with an Updated List of Currently Accepted Taxa. Plants 2021, 10, 1839. https://doi.org/10.3390/ plants10091839

Academic Editor: Daniel Potter

Received: 11 August 2021

Accepted: 1 September 2021

Published: 4 September 2021

Publisher's Note: MDPI stays neutral with regard to jurisdictional claims in published maps and institutional affiliations.

Copyright: (c) 2021 by the authors. Licensee MDPI, Basel, Switzerland. This article is an open access article distributed under the terms and conditions of the Creative Commons Attribution (CC BY) license (https:/ / creativecommons.org/licenses/by/ $4.0 /)$.

\begin{abstract}
The Epipactis helleborine (L.) Crantz group is one of the most taxonomically challenging species complexes within the genus Epipactis. Because of the exceptionally high levels of morphological variability and the ability to readily cross with other species, ninety different taxa at various taxonomic ranks have already been described within its nominative subspecies, but the taxonomic status of most of them is uncertain, widely disputed, and sometimes even irrelevant. The present review is based on results of the most recent research devoted to the E. helleborine group taxonomy. In addition, we analysed data about taxa belonging to this group presented in some research articles and monographs devoted directly to the genus Epipactis or to orchids in certain area(s). Based on the reviewed literature and data collected in four taxonomic databases Available Online, we propose an updated list of the 10 currently accepted taxa in the E. helleborine group (two species, six subspecies, and two varieties), which includes E. helleborine (L.) Crantz subsp. helleborine; E. helleborine subsp. bithynica (Robatsch) Kreutz; E. helleborine subsp. distans (Arv.-Touv.) R.Engel and P.Quentin; E. helleborine subsp. neerlandica (Verm.) Buttler; E. helleborine var. tangutica (Schltr.) S.C.Chen and G.H.Zhu; E. helleborine subsp. tremolsii (Pau) E.Klein; E. helleborine subsp. voethii (Robatsch) Jakubska-Busse, Żołubak, and Łobas, stat. nov.; E. condensata Boiss. ex D.P.Young; E. condensata var. kuenkeleana (Akhalk., H.Baumann, R.Lorenz, and Mosul.) Popovich; and E. cupaniana C.Brullo, D’Emerico, and Pulv.
\end{abstract}

Keywords: Epipactis; Helleborines; morphological species complex; orchids; taxonomy

\section{Introduction}

The long and turbulent history of changes in taxonomy of the genus Epipactis Zinn, 1757 (Orchidaceae) is well documented [1-12]. The most widely contested aspect of its taxonomy is whether to treat many of its morphologically different but poorly defined taxa as new species or if is more appropriate to transfer some of them to lower taxonomic ranks, such as subspecies, variety, or form $[13,14]$. The currently adopted classification system of Epipactis does not take sufficient account of the variation range of morphological characters within its taxa, nor does it explain the underlying sources of this variability. Moreover, the species delimitation within Epipactis is often complicated by the ease with which the interspecific hybrids are formed in the locations inside the native ranges of the parental species, the existence of which is a common cause of taxa misidentification [15-21]. The fluidity of the morphological boundaries between various Epipactis species causes serious difficulties in determinig the diagnostic characters useful in species identification [22-24]. As a result, there is still no official account of the species included within the genus Epipactis. The estimates range from a few to several dozen depending on the source (e.g., [6,17,25-38]).

The primary aim of taxonomic research is to provide a comprehensive classification system, which reflects the observed relationships between the taxa at the morphological, 
geographical, and genetic levels [39]. The major impediment in achieving a taxonomic consensus within the genus Epipactis is its exceptionally high morphological variability, which is still insufficiently documented and requires further clarification [11,14]. The main source of this variability, referred to the phenotypic plasticity, is manifested in natural populations of many Epipactis species through the slight differences observed in the vegetative (e.g., shoots and leaves) and generative (i.e., flowers and their separate elements) parts of the individual plants $[4,15,40-42]$. This broad spectrum of morphological variation also provided a wide range of characters that delimitate and group the species within the genus Epipactis over the past few decades (e.g., [14,17,21,23,28-32,43-54]). As a consequence, a large number of morphologically similar species and infraspecific taxa (i.e., subspecies, varieties, or forms), usually of local or narrowly restricted occurrence, have been described within Epipactis $[17,23,31,55]$. However, the taxonomy and systematic position of the majority of these taxa are still not well understood and highly debated [4,11,13,24,42,56-59].

One of these taxonomically problematic species is Epipactis helleborine (L.) Crantz, native to Eurasia and North Africa and widely naturalised in North America [27,31,32,58,60]. It is a common cross-pollinating (allogamous) orchid species with a very wide ecological amplitude. It grows in areas with nutrient rich soils and a broad $\mathrm{pH}$ spectrum (usually in alkaline conditions) and, unlike the other species of Epipactis, has a highly variable habitat preference. Its natural populations are usually found in forests, amongst shrubs, or in partly disturbed vegetation sites, ranging from lowland floodplains to mountain spruce forests up to the altitude of $2200 \mathrm{~m}$ a.s.1. [31,37,51-53,61-63]. It is also increasingly observed in the areas strongly impacted by human activity, such as roadsides, cemeteries, railway embankments, gravel pits, gardens, and urban parks [64,65].

The recently increased interest in the evolutionary history of the genus Epipactis has resulted in some significant changes in its taxonomy [11]. The most important and widely challenged one of them is considered the present division of this genus into nine morphologically distinct species complexes [66-69]. Among them, there is also a group devoted to E. helleborine, for which the circumscription has already been reorganized by numerous scientists $[3,5,8,10,17,23,30,31,47,70,71]$. Interestingly, the taxonomic status of individual taxa included in this group is still chaotic and in need of clarification.

Because of the general confusion concerning the taxonomy of the genus Epipactis, caused mainly by the frequent changes in its infrageneric classification, we aimed to present here an updated list of the 10 currently accepted taxa included in the E. helleborine group. As a decisive criterion for the selection of individual taxa to our circumscription, we used the results of recent genetic and morphometric analysis in relation to the total 41 taxa that have been included in this group.

\section{Recent Taxonomic Publications Devoted to the Epipactis helleborine Group}

So far, a number of the research articles and monographs have been published by representing the description and taxonomic treatments of taxa of the Epipactis helleborine group $[3,5,8,10,17,23,30,31,47,70,71]$ (see Table 1). At the beginning, Tyteca and Dufrêne [47] conducted the medium-scale biostatistical study of the genus Epipactis focused on only seven allogamous species (autogamous taxa were explicitly excluded) from the southwestern limit of its distribution range in Europe. But the authors concluded that at least five species (i.e., E. helleborine s.str., E. distans Arv.-Touv., E. neerlandica (Verm.) DevillersTersch. and Devillers, E. tremolsii Pau, and E. lusitanica D.Tyteca) should be included within the E. helleborine group. Tyteca and Dufrêne [47] also used the results of multivariate analysis of 28 carefully chosen characters of floral and vegetative morphology (particularly the differences in the flower structure) to prove that the four taxa included in this group are sufficiently different from E. helleborine s.str. and should be treated as independent species rather than at the subspecific rank.

Later, the circumscription of the Epipactis helleborine group was delimited by a yet another set of clearly defined morphological characters, including the appearance of the shoot, labellum, ovary, and pedicel. However, the morphometric analysis of these charac- 
ters was not as detailed as that of the other published taxonomic treatments (e.g., [23,47]). Delforge [30] divided the 23 species belonging to the E. helleborine group into three subgroups, i.e., the E. leptochila subgroup (five taxa), the E. helleborine subgroup (13 taxa), and the E. tremolsii subgroup (five taxa). Within the E. helleborine subgroup, this author included E. helleborine s.str. and 12 other morphologically similar species (Table 1). This subgroup was also further sub-divided into two additional sections: one with the cross-pollinating species and another with autogamous taxa only.

The increase in number of new taxa described within the genus Epipactis has led to some significant changes in its infrageneric classification. As a consequence, two new characters were added by Delforge [17] to the circumscription of the E. helleborine group, i.e., the leaf and the inflorescence morphology. Four of the previously used characters, i.e., the appearance of the shoot, labellum, ovary, and pedicel have also been redefined. Thus, Delforge's newly circumscribed E. helleborine group included 13 taxa (11 at the rank of species and two varieties). Six of which were included in the author's previous study [30], where one (i.e., E. helleborine var. youngiana A.J.Richards and A.F.Porter) Kreutz) has changed its taxonomic rank (Table 1 ).

Subsequently, Brullo et al. [23] have expanded the Epipactis helleborine group by including E. cupaniana C.Brullo, D'Emerico, and Pulv., a newly described endemic from the mesophilous Holm oak woods in north-central Sicily. Their circumscription of the E. helleborine group included 11 additional species and was broadly based on the system proposed by Delforge in 2006 (Table 1). The authors also conducted a morphometric analysis of a broad range of 37 characters of floral and vegetative morphology. The obtained results suggest that E. cupaniana does indeed belong to the E. helleborine group. This taxon is morphologically and karyologically different from E. helleborine s.str. and can be accepted as a separate species. Despite this conclusion, Delforge [31] did not include E. cupaniana in his latest concept of the E. helleborine group.

One year later, in 2014, Epipactis condensata subsp. kuenkeleana (Akhalk., H.Baumann, R.Lorenz, and Mosul.) Kreutz, Fateryga, and Efimov was published as a new combination for the species formerly known as E. viridiflora subsp. kuenkeleana Akhalk., H.Baumann, R.Lorenz, and Mosul. [8], where then Delforge raised this latter taxon to full species status (i.e., E. kuenkeleana (Akhalk., H.Baumann, R.Lorenz and Mosul.) P.Delforge) [72]. Thereafter, E. condensata subsp. kuenkeleana was put into synonymy with the nominative subspecies [10,70]. However, finally, in 2020, plants within the same taxon corresponding to the former subsp. kuenkeleana were considered as a phenotypic form, confined to shady forest communities, and described as E. condensata var. kuenkeleana (Akhalk., H.Baumann, R.Lorenz, and Mosul.) Popovich [70].

The most recent taxonomic treatment of the Epipactis helleborine group in Europe, North Africa, and the Middle East [31], is broadly based on an earlier account by the same author [17] and expands to comprise 17 taxa, five of which are included here for the first time, and one taxon (i.e., E. pontica Taubenheim) which is transferred to E. leptochila group (Table 1). 
Table 1. Nomenclatural and taxonomic changes within the Epipactis helleborine group.

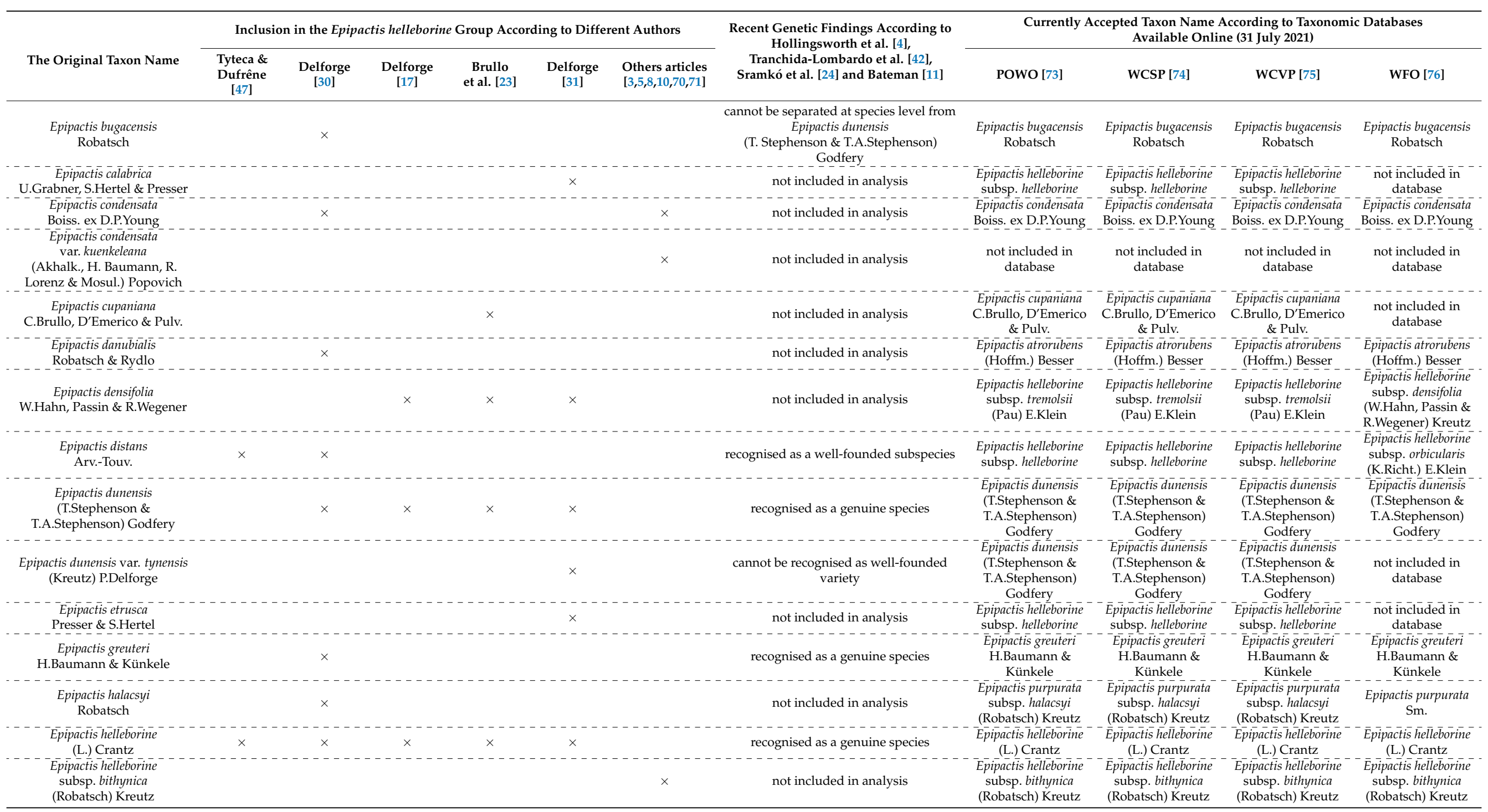


Table 1. Cont.

\begin{tabular}{|c|c|c|c|c|c|c|c|c|c|c|c|}
\hline \multirow[b]{2}{*}{ The Original Taxon Name } & \multicolumn{6}{|c|}{ Inclusion in the Epipactis helleborine Group According to Different Authors } & \multirow{2}{*}{$\begin{array}{l}\text { Recent Genetic Findings According to } \\
\text { Hollingsworth et al. [4], } \\
\text { Tranchida-Lombardo et al. [42], } \\
\text { Sramkó et al. [24] and Bateman [11] }\end{array}$} & \multicolumn{4}{|c|}{$\begin{array}{c}\text { Currently Accepted Taxon Name According to Taxonomic Databases } \\
\text { Available Online (31 July 2021) } \\
\end{array}$} \\
\hline & $\begin{array}{c}\text { Tyteca \& } \\
\text { Dufrêne } \\
\text { [47] }\end{array}$ & $\begin{array}{l}\text { Delforge } \\
{[30]}\end{array}$ & $\begin{array}{c}\text { Delforge } \\
{[17]}\end{array}$ & $\begin{array}{l}\text { Brullo } \\
\text { et al. [23] }\end{array}$ & $\begin{array}{c}\text { Delforge } \\
{[31]}\end{array}$ & $\begin{array}{l}\text { Others articles } \\
{[3,5,8,10,70,71]}\end{array}$ & & POWO [73] & WCSP [74] & WCVP [75] & WFO [76] \\
\hline $\begin{array}{c}\text { Epipactis helleborine } \\
\text { var. castanearum } \\
\text { Gévaudan, Nicole \& Anglade }\end{array}$ & & & & & $x$ & & not included in analysis & $\begin{array}{l}\text { Epipactis helleborine } \\
\text { subsp. tremolsii } \\
\text { (Pau) E.Klein }\end{array}$ & $\begin{array}{l}\text { Epipactis helleborine } \\
\text { subsp. tremolsii } \\
\text { (Pau) E.Klein }\end{array}$ & $\begin{array}{l}\text { Epipactis helleborine } \\
\text { subsp. tremolsii } \\
\text { (Pau) E.Klein }\end{array}$ & $\begin{array}{l}\text { Epipactis helleborine } \\
\text { (L.) Crantz }\end{array}$ \\
\hline $\begin{array}{l}\text { Epipactis helleborine } \\
\text { var. orbicularis } \\
\text { (C.Richt) Soó }\end{array}$ & & & $\times$ & & $\times$ & & not included in analysis & $\begin{array}{l}\text { not included in } \\
\text { database }\end{array}$ & $\begin{array}{l}\text { Epipactis helleborine } \\
\text { subsp. helleborine }\end{array}$ & $\begin{array}{l}\text { not included in } \\
\text { database }\end{array}$ & $\begin{array}{l}\text { Epipactis helle éborine- } \\
\text { subsp. orbicularis } \\
\text { (K.Richt) E.Klein }\end{array}$ \\
\hline 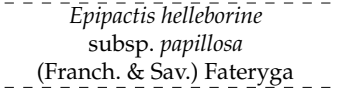 & & & & & & $\times$ & not included in analysis & $\begin{array}{l}\text { Epipactis papillosa } \\
\text { Franch. \& Sav. }\end{array}$ & $\begin{array}{l}\text { Epipactis papillosa } \\
\text { Franch. \& Sav. }\end{array}$ & $\begin{array}{l}\text { Epipacti papillosa } \\
\text { Franch. \& Sav. }\end{array}$ & $\begin{array}{l}\text { not included in } \\
\text { database }\end{array}$ \\
\hline $\begin{array}{l}\text { Epipactis helleborine } \\
\text { var. tangutica } \\
\text { (Schltr.) S.C.Chen \& G.H.Zhu }\end{array}$ & & & & & & $\times$ & not included in analysis & $\begin{array}{l}\text { Epipactis helléborine } \\
\text { var. tangutica } \\
\text { (Schltr.) S.C.Chen \& } \\
\text { G.H.Zhu }\end{array}$ & 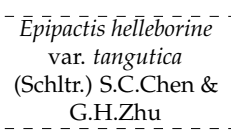 & $\begin{array}{l}\text { Epipactīis hêllèerorine- } \\
\text { var. tangutica } \\
\text { (Schltr.) S.C.Chen \& } \\
\text { G.H.Zhu }\end{array}$ & 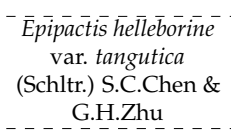 \\
\hline $\begin{array}{l}\text { Epipactis hellétoborine-- } \\
\text { var. youngiana } \\
\text { (A.J.Richards \& } \\
\text { A.F.Porter) Kreutz }\end{array}$ & & & $\times$ & & $\times$ & & $\begin{array}{l}\text { synonymised with Epipactis helleborine } \\
\text { subsp. neerlandica } \\
\text { (Verm.) Buttler }\end{array}$ & $\begin{array}{l}\text { Epipactis helleborine } \\
\text { subsp. neerlandica } \\
\text { (Verm.) Buttler }\end{array}$ & $\begin{array}{l}\text { Epipactis helleborine } \\
\text { subsp. neerlandica } \\
\text { (Verm.) Buttler }\end{array}$ & $\begin{array}{l}\text { Epipactis helleborine } \\
\text { subsp. neerlandica } \\
\text { (Verm.) Buttler }\end{array}$ & $\begin{array}{l}\text { Epipactis helleborine } \\
\text { (L.) Crantz }\end{array}$ \\
\hline $\begin{array}{l}\text { Epipactis heraclea } \\
\text { P.Delforge \& Kreutz }\end{array}$ & & & $\times$ & $x$ & $\times$ & & not included in analysis & $\begin{array}{l}\text { Epipactiti helleborine } \\
\text { subsp. tremolsii } \\
\text { (Pau) E.Klein }\end{array}$ & $\begin{array}{l}\text { Épipactis helile } \bar{b} \text { orine } \\
\text { subsp. tremolsii } \\
\text { (Pau) E.Klein }\end{array}$ & $\begin{array}{l}\text { Epipactis hellèborine- } \\
\text { subsp. tremolsii } \\
\text { (Pau) E.Klein }\end{array}$ & $\begin{array}{l}\text { Epipactis heraclea } \\
\text { P.Delforge \& Kreutz }\end{array}$ \\
\hline $\begin{array}{l}\text { (W.Rossi \& E.Klein) B.Baumann } \\
\text { \& H.Baumann } \\
\text { Epipactis leptótochila } \\
\text { (Godfery) Godfery }\end{array}$ & & $x$ & & & & & $\begin{array}{l}\text { not included in analysis } \\
\text { recognised as a genuine species }\end{array}$ & 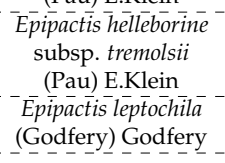 & $\begin{array}{l}\text { Epipactis helle } \bar{c} \text { orine } \\
\text { subsp. tremolsii } \\
\text { (Pau) E.Klein } \\
\text { Epipactis le leptochitia } \\
\text { (Godfery) Godfery }\end{array}$ & 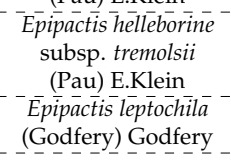 & $\begin{array}{l}\text { Epipactis helle éborine- } \\
\text { subsp. latina } \\
\text { W.Rossi \& E.Klein } \\
\text { Epipactis leptochila } \\
\text { Epodfery) Godfery }\end{array}$ \\
\hline $\begin{array}{l}\text { Epipactis leutei } \\
\text { Robatsch }\end{array}$ & & $\times$ & & & & & not included in analysis & $\begin{array}{l}\text { Epipactis helleborine } \\
\text { subsp. helleborine }\end{array}$ & $\begin{array}{l}\text { Epipactis helleborine } \\
\text { subsp. helleborine }\end{array}$ & $\begin{array}{l}\text { Epipactis helleborine } \\
\text { subsp. helleborine }\end{array}$ & $\begin{array}{l}\text { Epipactis helleboriñe } \\
\text { subsp. leutei } \\
\text { (Robatsch) Kreutz }\end{array}$ \\
\hline $\begin{array}{l}-{ }^{-} \text {Epipactis levantina } \\
\text { (Kreutz, Óvári \& Shifman) } \\
\text { P.Delforge }\end{array}$ & & & & & $\times$ & & not included in analysis & $\begin{array}{l}\text { Epipactis helleborine } \\
\text { subsp. tremolsii } \\
\text { (Pau) E.Klein }\end{array}$ & $\begin{array}{l}\text { Epipactis helleborine- } \\
\text { subsp. tremolsii } \\
\text { (Pau) E.Klein }\end{array}$ & $\begin{array}{l}\text { Epipactis helleborine } \\
\text { subsp. tremolsii } \\
\text { (Pau) E.Klein }\end{array}$ & $\begin{array}{l}\text { not included in } \\
\text { database }\end{array}$ \\
\hline $\begin{array}{l}\text { Epipactis lusitanica } \\
\text { D.Tyteca }\end{array}$ & $\times$ & $\times$ & & & & & $\begin{array}{l}\text { cannot beparated at species level from } \\
\text { recognised as arguably } \\
\text { synonymous with } \\
\text { Epipactis helleborine s.str. }\end{array}$ & $\begin{array}{l}\text { Epipactis helleborine } \\
\text { subsp. tremolsii } \\
\text { (Pau) E.Klein }\end{array}$ & $\begin{array}{l}\text { Epipactis helleborine } \\
\text { subsp. tremolsii } \\
\text { (Pau) E.Klein }\end{array}$ & $\begin{array}{l}\text { Epipactis helleborine } \\
\text { subsp. tremolsii } \\
\text { (Pau) E.Klein }\end{array}$ & $\begin{array}{l}\text { Epipactis lusitanica } \\
\text { D.Tyteca }\end{array}$ \\
\hline $\begin{array}{l}\text { Epipactis meridionalis } \\
\text { H.Baumann \& R.Lorenz }\end{array}$ & & $\times$ & $\times$ & $\times$ & $\times$ & & $\begin{array}{c}\text { cannot be separated at species level from } \\
\text { Epipactis helleborine s.str;; }\end{array}$ & $\begin{array}{l}\text { Epipactis helleborine } \\
\text { subsp. helleborine }\end{array}$ & $\begin{array}{l}\text { Epipactis helleborine } \\
\text { subsp. helleborine }\end{array}$ & $\begin{array}{l}\text { Epipactis helleborine } \\
\text { subsp. helleborine }\end{array}$ & $\begin{array}{l}\text { Epipactis meridionalis } \\
\text { H.Baumann \& } \\
\text { R.Lorenz }\end{array}$ \\
\hline $\begin{array}{l}\text { Epipactis molochina } \\
\text { P.Delforge }\end{array}$ & & & $\times$ & $\times$ & $\times$ & & not included in analysis & $\begin{array}{l}\text { Epipactis helleborine } \\
\text { subsp. helleborine }\end{array}$ & $\begin{array}{l}\text { Epipactis helleborine } \\
\text { subsp. helleborine }\end{array}$ & $\begin{array}{l}\text { Epipactis helleborine } \\
\text { subsp. helleborine }\end{array}$ & 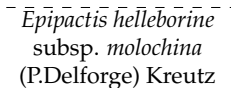 \\
\hline $\begin{array}{l}\text { Epipactis mùuêleri } \\
\text { Godfery }\end{array}$ & & $x$ & & & & & recognised as a genuine species & $\begin{array}{l}\text { - Épipactis muèileri } \\
\text { Godfery }\end{array}$ & $\begin{array}{l}\text { Epipactis muelleri } \\
\text { Godfery }\end{array}$ & $\begin{array}{l}\text { Epipacti muelleri } \\
\text { Godfery }\end{array}$ & $\begin{array}{c}\text { Epipactis muelleri } \\
\text { Godfery }\end{array}$ \\
\hline $\begin{array}{l}\text { Epipactis naousaensis } \\
\quad \text { Robatsch }\end{array}$ & & $\times$ & & & & & $\begin{array}{c}\text { cannot be separated at species level from } \\
\text { Epipactis helleborine s.str. }\end{array}$ & $\begin{array}{l}\text { Epipactis greuteri } \\
\text { H.Baumann \& } \\
\text { Künkele }\end{array}$ & $\begin{array}{l}\text { Epipactis greuteri } \\
\text { H.Baumann \& } \\
\text { Künkele }\end{array}$ & $\begin{array}{l}\text { Epipactis greuteri } \\
\text { H.Baumann \& } \\
\text { Künkele }\end{array}$ & $\begin{array}{l}\text { Epipactis leptochila } \\
\text { subsp. naousaensis } \\
\text { (Robatsch) Kreutz }\end{array}$ \\
\hline
\end{tabular}


Table 1. Cont.

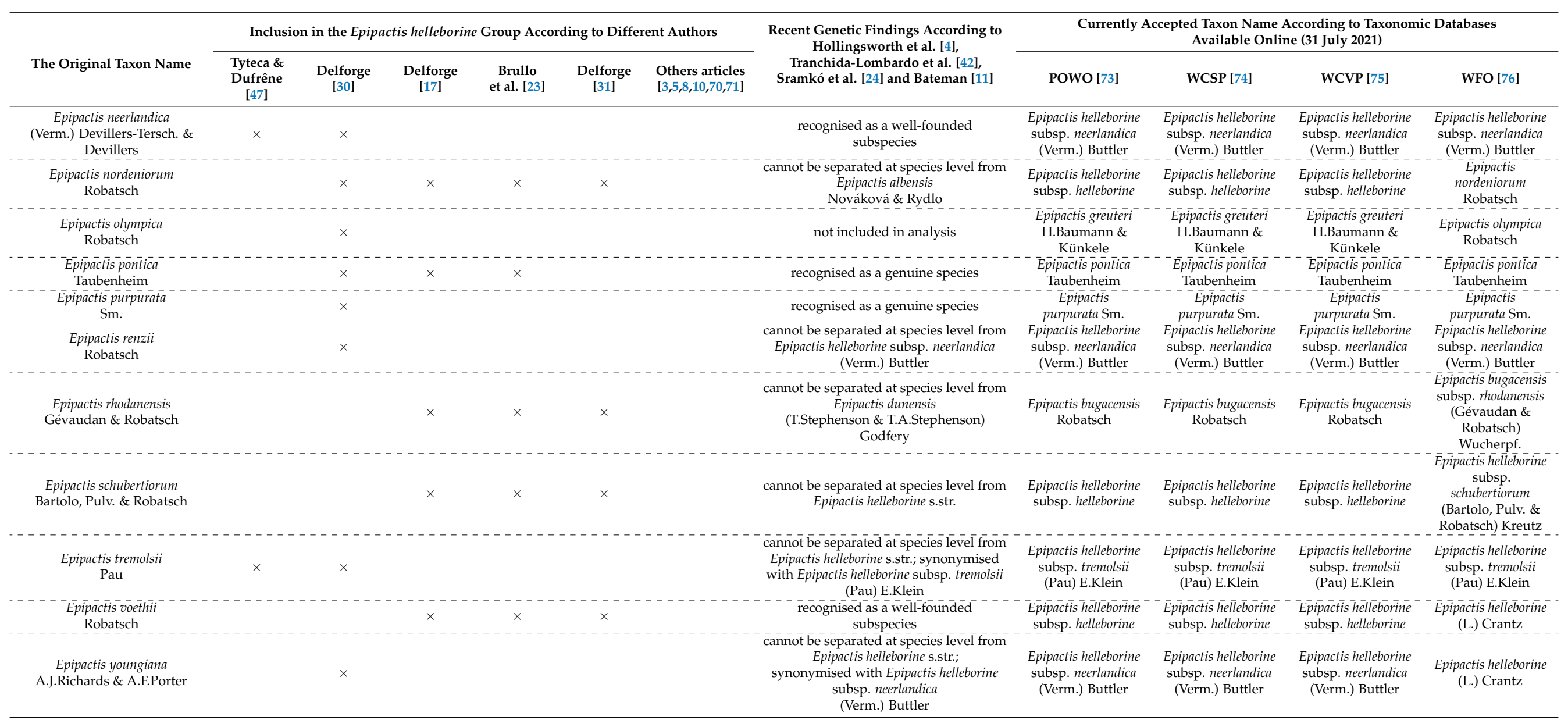




\section{List of Names of Infraspecific Taxa in Epipactis helleborine and its Current Taxonomic Status}

The seemingly endless morphological variation observed across the entire distribution range of Epipactis helleborine s.str. is clearly reflected by the list of its infraspecific taxa presented below in Table 2.

Table 2. An overview of names of infraspecific taxa published in Epipactis helleborine.

\begin{tabular}{|c|c|c|}
\hline $\begin{array}{l}\text { The Name and Its Infraspecific Rank in } \\
\text { Epipactis helleborine }\end{array}$ & $\begin{array}{l}\text { The Original Name and the Year } \\
\text { of Its Publication }\end{array}$ & $\begin{array}{l}\text { Currently Accepted Name } \\
\text { (31 July 2021)* }\end{array}$ \\
\hline $\begin{array}{l}\text { E. helleborine subsp. aspromontana (Bartolo, Pulv. \& Robatsch) } \\
\text { H.Baumann \& R.Lorenz }\end{array}$ & E. aspromontana Bartolo, Pulv. \& Robatsch (1996) & E. helleborine subsp. helleborine \\
\hline E. helleborine subsp. bithynica (Robatsch) Kreutz & E. bithynica Robatsch (1991) & E. helleborine subsp. bithynica (Robatsch) Kreutz \\
\hline E. helleborine subsp. condensata (Boiss. ex D.P.Young) H.Sund. & E. condensata Boiss. ex D.P.Young (1970). & E. condensata Boiss. ex D.P.Young \\
\hline E. helleborine subsp. confusa (D.P.Young) H.Sund. & E. confusa D.P.Young (1953) & E. phyllanthes G.E.Sm. \\
\hline E. helleborine subsp. degenii (Szentp. \& Mónus) Kreutz & E. degenii Szentp. \& Mónus (1999) & E. helleborine subsp. helleborine \\
\hline E. helleborine subsp. densifolia (W.Hahn, Passin \& R.Wegener) Kreutz & E. densifolia W.Hahn, Passin \& R.Wegener (2003) & E. helleborine subsp. tremolsii (Pau) E.Klein \\
\hline E. helleborine subsp. distans (Arv.-Touv.) R.Engel \& P.Quentin & E. distans Arv.-Touv. (1873) & E. helleborine subsp. helleborine \\
\hline E. helleborine subsp. helleborine & E. helleborine (L.) Crantz (1769) & E. helleborine subsp. helleborine \\
\hline E. helleborine subsp. latifolia (L.) Syme & Serapias helleborine var. latifolia L. (1753) & E. helleborine subsp. helleborine \\
\hline E. helleborine subsp. latina W.Rossi \& E.Klein & E. helleborine subsp. latina W.Rossi \& E.Klein (1987) & E. helleborine subsp. tremolsii (Pau) E.Klein \\
\hline E. helleborine subsp. leptochila (Godfery) Soó & E. viridiflora var. leptochila Godfery (1919) & E. leptochila (Godfery) Godfery \\
\hline E. helleborine subsp. leutei (Robatsch) Kreutz & E. leutei Robatsch (1989) & E. helleborine subsp. helleborine \\
\hline E. helleborine subsp. levantina Kreutz & E. helleborine subsp. tremolsii (Pau) E.Klein (1979) & E. helleborine subsp. tremolsii (Pau) E.Klein \\
\hline E. helleborine subsp. lusitanica (D.Tyteca) J.-M.Tison & E. lusitanica D.Tyteca (1988) & E. helleborine subsp. tremolsii (Pau) E.Klein \\
\hline E. helleborine subsp. minor (R.Engel) R.Engel & E. helleborine var. minor R.Engel (1994) & E. helleborine subsp. helleborine \\
\hline E. helleborine subsp. molochina (P.Delforge) Kreutz & E. molochina P.Delforge (2004) & E. helleborine subsp. helleborine \\
\hline E. helleborine subsp. moratoria Riech. \& Zirnsack & E. helleborine subsp. moratoria Riech. \& Zirnsack (2008) & E. helleborine subsp. helleborine \\
\hline E. helleborine subsp. muelleri (Godfery) O.Bolòs, Masalles \& Vigo & E. muelleri Godfery (1921) & E. muelleri Godfery \\
\hline E. helleborine subsp. neerlandica (Verm.) Buttler & E. helleborine var. neerlandica Verm. (1949) & E. helleborine subsp. neerlandica (Verm.) Buttler \\
\hline E. helleborine subsp. ohwii (Fukuy.) H.J.Su & E. ohwii Fukuy. (1934) & E. ohwii Fukuy. \\
\hline E. helleborine subsp. orbicularis (K.Richt.) E.Klein & E. orbicularis K.Richt. (1887) & E. helleborine subsp. helleborine \\
\hline E. helleborine subsp. papillosa (Franch. \& Sav.) Fateryga & E. papillosa Franch. \& Sav. (1878) & E. papillosa Franch. \& Sav. \\
\hline E. helleborine subsp. persica (Soó) H.Sund. & Helleborine persica Soó (1927) & E. persica (Soó) Hausskn. ex Nannf. \\
\hline E. helleborine subsp. phyllanthes (G.E.Sm.) H.Sund. & E. phyllanthes G.E.Sm. (1852) & E. phyllanthes G.E.Sm. \\
\hline E. helleborine subsp. pontica (Taubenheim) H.Sund. & E. pontica Taubenheim (1975) & E. pontica Taubenheim \\
\hline E. helleborine subsp. renzii (Robatsch) Løjtnant & E. renzii Robatsch (1988) & E. helleborine subsp. neerlandica (Verm.) Buttler \\
\hline E. helleborine subsp. schubertiorum (Bartolo, Pulv. \& Robatsch) Kreutz & E. schubertiorum Bartolo, Pulv. \& Robatsch (1997) & E. helleborine subsp. helleborine \\
\hline E. helleborine subsp. transcaucasica A.P.Khokhr. & E. helleborine subsp. transcaucasica A.P.Khokhr. (1991) & E. persica (Soó) Hausskn. ex Nannf. \\
\hline E. helleborine subsp. tremolsii (Pau) E.Klein & E. tremolsii Pau (1914) & E. helleborine subsp. tremolsii (Pau) E.Klein \\
\hline E. helleborine subsp. troodi (H.Lindb.) H.Sund. & E. troodi H.Lindb. (1942) & E. persica (Soó) Hausskn. ex Nannf. \\
\hline E. helleborine subsp. turcica (Kreutz) Véla \& Viglione & E. turcica Kreutz (1997) & E. helleborine subsp. tremolsii (Pau) E.Klein \\
\hline E. helleborine subsp. varians (Crantz) H.Sund. & E. helleborine var. varians Crantz (1769) & E. purpurata $\mathrm{Sm}$. \\
\hline E. helleborine subsp. viridans (Crantz) O.Schwarz & E. helleborine var. viridans Crantz (1769) & E. atrorubens (Hoffm.) Besser \\
\hline E. helleborine subsp. viridiflora (Hoffm.) O.Schwarz & Serapias latifolia viridiflora Hoffm. (1804) & E. purpurata Sm. \\
\hline E. helleborine subsp. viridis Soó & E. helleborine subsp. viridis Soó (1969) & E. helleborine subsp. helleborine \\
\hline E. helleborine subsp. zirnsackiana (Riech.) Riech. & E. zirnsackiana Riech. (2010) & E. helleborine subsp. helleborine \\
\hline E. helleborine var. canescens (Irmisch) Rchb.f. & E. microphylla var. canescens Irmisch (1846) & E. microphylla (Ehrh.) Sw. \\
\hline E. helleborine var. castanearum Gévaudan, Nicole \& Anglade & $\begin{array}{l}\text { E. helleborine var. castanearum Gévaudan, Nicole \& } \\
\text { Anglade (2011) }\end{array}$ & E. helleborine subsp. tremolsii (Pau) E.Klein \\
\hline E. helleborine var. chlorantha Verm. & E. helleborine var. chlorantha Verm. (1949) & E. helleborine subsp. helleborine \\
\hline E. helleborine var. densiflora Verm. & E. helleborine var. densiflora Verm. (1949) & E. helleborine subsp. helleborine \\
\hline E. helleborine var. diversifolia Verm. & E. helleborine var. diversifolia Verm. (1949) & E. helleborine subsp. helleborine \\
\hline E. helleborine var. herbacea (Lindl.) S.N.Mitra & E. herbacea Lindl. (1839) & E. helleborine subsp. helleborine \\
\hline E. helleborine var. interrupta Beck & E. helleborine var. interrupta Beck (1890) & E. helleborine subsp. helleborine \\
\hline E. helleborine var. intrusa (Lindl.) S.N.Mitra & E. intrusa Lindl. (1857) & E. helleborine subsp. helleborine \\
\hline E. helleborine var. lancifolia (Zapal.) Bordz. & E. viridans var. lancifolia Zapal. (1906) & E. helleborine subsp. helleborine \\
\hline E. helleborine var. latifolia (L.) A.Blytt & Serapias helleborine var. latifolia L. (1753) & E. helleborine subsp. helleborine \\
\hline E. helleborine var. laxiflora Verm. & E. helleborine var. laxiflora Verm. (1949) & E. helleborine subsp. helleborine \\
\hline E. helleborine var. microphylla (Ehrh.) Rchb.f. & Serapias microphylla Ehrh. (1789) & E. microphylla (Ehrh.) Sw. \\
\hline E. helleborine var. minor R.Engel & E. helleborine var. minor R.Engel (1994) & E. helleborine subsp. helleborine \\
\hline E. helleborine var. monotropoides (Mousley) L.Lewis & Amesia latifolia f. monotropoides Mousley (1927) & E. helleborine subsp. helleborine \\
\hline E. helleborine var. moratoria (Riech. \& Zirnsack) P.Delforge & E. helleborine subsp. moratoria Riech. \& Zirnsack (2008) & E. helleborine subsp. helleborine \\
\hline E. helleborine var. neerlandica Verm. & E. helleborine var. neerlandica Verm. (1949) & E. helleborine subsp. neerlandica (Verm.) Buttler \\
\hline E. helleborine var. nuda (Irmisch) Rchb.f. & E. microphylla var. nuda Irmisch (1846) & E. microphylla (Ehrh.) Sw. \\
\hline E. helleborine var. orbicularis (C. Richt) Soó & E. orbicularis C.Richt. (1887) & E. helleborine subsp. helleborine \\
\hline E. helleborine var. orbicularis (K.Richt.) Verm. & E. orbicularis K.Richt. (1887) & E. helleborine subsp. helleborine \\
\hline E. helleborine var. pallens Gaudin & E. helleborine var. pallens Gaudin (1829) & E. helleborine subsp. helleborine \\
\hline E. helleborine var. papillosa (Franch. \& Sav.) T.Hashim. & E. papillosa Franch. \& Sav. (1878) & E. papillosa Franch. \& Sav. \\
\hline E. helleborine var. phoenicea Verm. & E. helleborine var. phoenicea Verm. (1949) & E. helleborine subsp. helleborine \\
\hline E. helleborine var. rectilinguis Murb. & E. helleborine var. rectilinguis Murb. (1891) & E. leptochila (Godfery) Godfery \\
\hline E. helleborine var. renzii (Robatsch) J.Claess. Kleynen \& Wielinga & E. renzii Robatsch (1988) & E. helleborine subsp. neerlandica (Verm.) Buttler \\
\hline E. helleborine var. rubiginosa Crantz & E. helleborine var. rubiginosa Crantz (1769) & E. atrorubens (Hoffm.) Besser \\
\hline E. helleborine var. sayekiana (Makino) T.Hashim. & E. sayekiana Makino (1918) & E. papillosa Franch. \& Sav. \\
\hline E. helleborine var. tangutica (Schltr.) S.C.Chen \& G.H.Zhu & E. tangutica Schltr. (1919) & E. helleborine var. tangutica (Schltr.) S.C.Chen \& G.H.Zhu \\
\hline E. helleborine var. thomsonii (Hook. f.) Aswal & E. latifolia var. thomsonii Hook.f. (1890) & E. helleborine subsp. helleborine \\
\hline E. helleborine var. thomsonii (Hook. f.) Karthik. & E. latifolia var. thomsonii Hook.f. (1890) & E. helleborine subsp. helleborine \\
\hline E. helleborine var. thomsonii (Hook. f.) R.R. Stewart & E. latifolia var. thomsonii Hook.f. (1890) & E. helleborine subsp. helleborine \\
\hline E. helleborine var. thomsonii (Hook.f.) S.N.Mitra & E. latifolia var. thomsonii Hook.f. (1890) & E. helleborine subsp. helleborine \\
\hline E. helleborine var. varians Crantz & E. helleborine var. varians Crantz (1769) & E. purpurata $\mathrm{Sm}$. \\
\hline E. helleborine var. violacea (Dur-Doq.) Rchb.f. & E. latifolia var. violacea Dur-Duq. (1846) & E. purpurata Sm. \\
\hline E. helleborine var. viridans Crantz & E. helleborine var. viridans Crantz (1769) & E. atrorubens (Hoffm.) Besser \\
\hline E. helleborine var. viridens A.Gray & E. helleborine var. viridens A.Gray (1879) & E. helleborine subsp. helleborine \\
\hline E. helleborine var. viridiflora (Hoffm.) Bordz. & Serapias latifolia viridiflora Hoffm. (1804) & E. purpurata $\mathrm{Sm}$. \\
\hline E. helleborine var. youngiana (A.J. Richards \& A.F.Porter) Kreutz & E. youngiana A.J.Richards \& A.F.Porter (1982) & E. helleborine subsp. neerlandica (Verm.) Buttler \\
\hline
\end{tabular}


Table 2. Cont.

\begin{tabular}{|c|c|c|}
\hline $\begin{array}{c}\text { The Name and Its Infraspecific Rank in } \\
\text { Epipactis helleborine }\end{array}$ & $\begin{array}{l}\text { The Original Name and the Year } \\
\text { of Its Publication }\end{array}$ & $\begin{array}{l}\text { Currently Accepted Name } \\
\text { (31 July 2021)* }\end{array}$ \\
\hline E. helleborine f. alba (Webster) B.Boivin & E. latifolia f. alba Webster (1898) & E. helleborine subsp. helleborine \\
\hline E. helleborine f. albifolia M.R.Lowe & E. helleborine f. albifolia M.R.Lowe (1990) & E. helleborine subsp. helleborine \\
\hline E. helleborine f. brevibracteata (Zapal.) Bordz. & E. viridans var. brevibracteata Zapal. (1906) & E. helleborine subsp. helleborine \\
\hline E. helleborine f. dentata (Zapal.) Soó & E. viridans var. dentata Zapal. (1906) & E. helleborine subsp. helleborine \\
\hline E. helleborine f. dilatata (Asch. \& Graebn.) Soó & E. latifolia var. dilatata Asch. \& Graebn. (1907) & E. helleborine subsp. helleborine \\
\hline E. helleborine f. gracilis (Dageforde ex Hegi) Pauca \& Morariu & E. latifolia f. gracilis Dageforde ex Hegi (1909) & E. albensis Nováková \& Rydlo \\
\hline E. helleborine f. helleborine & E. helleborine (L.) Crantz (1769) & E. helleborine subsp. helleborine \\
\hline E. helleborine f. luteola P.M.Br. & E. helleborine f. luteola P.M.Br. (1996) & E. helleborine subsp. helleborine \\
\hline E. helleborine f. macrophylla Snarskis & E. helleborine f. macrophylla Snarskis (1963) & E. helleborine subsp. helleborine \\
\hline E. helleborine f. minor (R.Engel) P.Delforge & E. helleborine var. minor R.Engel (1994) & E. helleborine subsp. helleborine \\
\hline E. helleborine f. monotropoides (Mousley) Scoggan & Amesia latifolia f. monotropoides Mousley (1927) & E. helleborine subsp. helleborine \\
\hline E. helleborine f. montana (Zapal.) Bordz. & E. viridans var. montana Zapal. (1906) & E. helleborine subsp. helleborine \\
\hline E. helleborine f. obtusa (Zapal.) Soó & E. viridans var. obtusa Zapal. (1906) & E. helleborine subsp. helleborine \\
\hline E. helleborine f. parviflora (Zapal.) Bordz. & E. viridans f. parviflora Zapal. (1906) & E. helleborine subsp. helleborine \\
\hline E. helleborine f. przemysliensis (Zapal.) Verm. & E. viridans var. przemysliensis Zapal. (1906) & E. helleborine subsp. helleborine \\
\hline E. helleborine f. remota (Zapal.) Bordz. & E. viridans f. remota Zapal. (1906) & E. helleborine subsp. helleborine \\
\hline E. helleborine f. variegata (Webster) B.Boivin & E. latifolia f. variegata Webster (1898) & E. helleborine subsp. helleborine \\
\hline
\end{tabular}

* According to [73-76].

As it turns out, in the light of the data collected in four taxonomic databases Available Online [73-76], as many as ninety morphologically similar taxa have been distinguished within Epipactis helleborine s.str. at various taxonomic ranks since its original description as Serapias helleborine L. by Carl Linnaeus in 1753 [33,77-82]. Among these, at the ranks of variety and subspecies have been classified respectively 37 and 36 names of taxa, and at the rank of form, 17 have been classified (Figure 1).

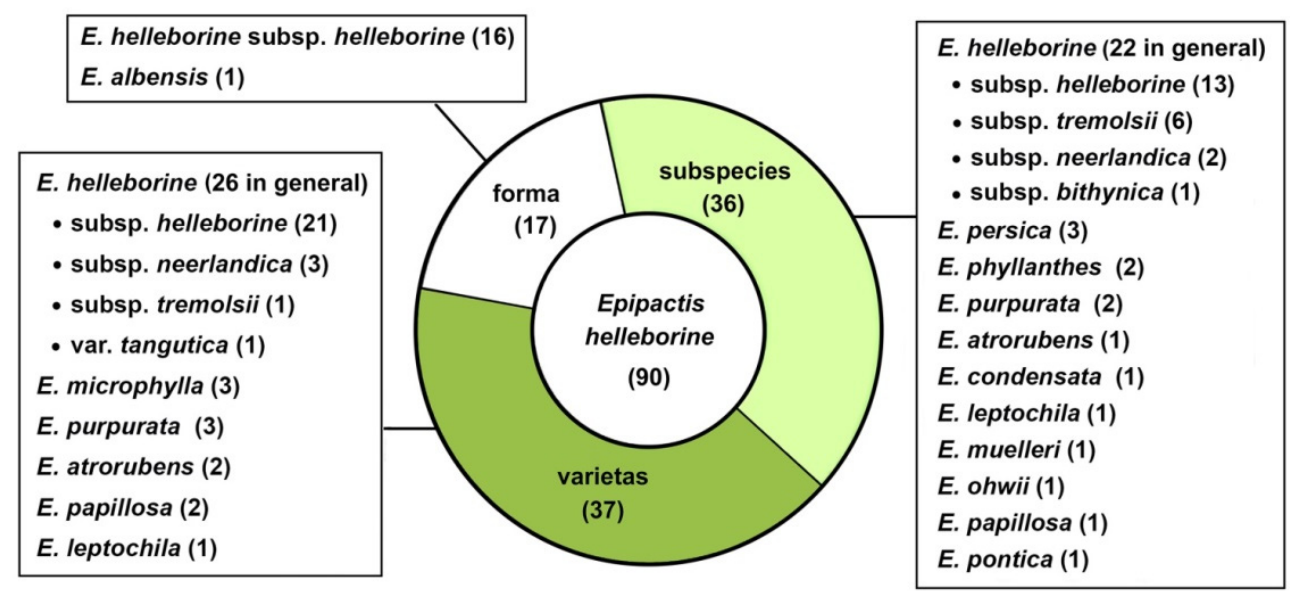

Figure 1. The current status of names of infraspecific taxa published in Epipactis helleborine categorised according to their taxonomic ranks, based on [73-76]. The number of names synonymised with E. helleborine s.l. and with other species is shown in parentheses.

It should be noted that most of them (64) were synonymised with Epipactis helleborine s.l. almost as soon as they were published and currently only five infraspecific taxa are accepted, i.e., E. helleborine (L.) Crantz subsp. helleborine, E. helleborine subsp. bithynica (Robatsch) Kreutz, E. helleborine subsp. neerlandica (Verm.) Buttler, E. helleborine var. tangutica (Schltr.) S.C.Chen and G.H.Zhu, and E. helleborine subsp. tremolsii (Pau) E.Klein $[3,63,71,83,84]$. The remaining 26 published names of taxa do not currently have a taxonomic relationship with E. helleborine s.l. (Figure 2). 


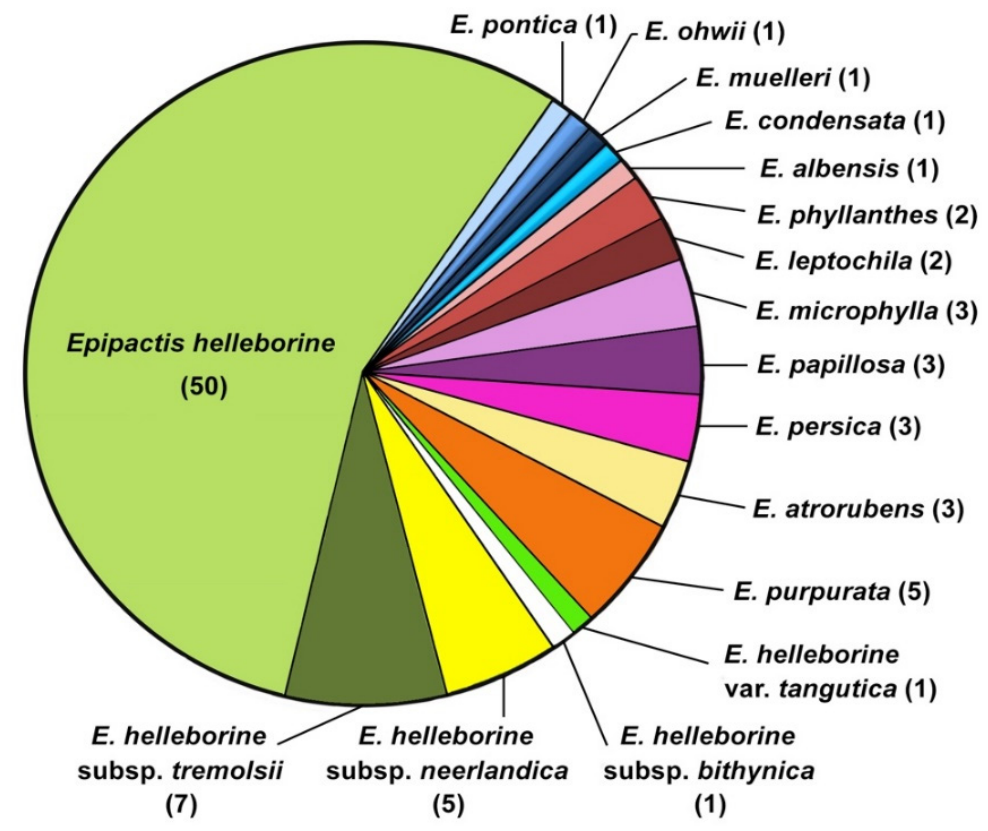

Figure 2. The current status of names of infraspecific taxa published in Epipactis helleborine, based on [73-76]. The number of names synonymised with E. helleborine s.l. and to other species is shown in parentheses.

\section{Conclusions}

Since the genus Epipactis has been divided into several species complexes based on morphological characters, more than forty taxa have been classified into the E. helleborine group (see Table 1). These contain such taxa as E. danubialis Robatsch and Rydlo, E. greuteri H.Baumann \& Künkele, E. halacsyi Robatsch, E. leptochila (Godfery) Godfery, E. muelleri Godfery, E. naousaensis Robatsch, E. olympica Robatsch, E. pontica or E. purpurata Sm., which, because of their distinct morphological phenotype, were excluded from it over time and (in some cases) provided a basis for effective distinguishing of other groups. Despite the fact that 15 of these taxa were originally included as separate species, they are being considered currently as three out of the five infraspecific taxa published in E. helleborine (i.e., E. helleborine subsp. helleborine, E. helleborine subsp. neerlandica, and E. helleborine subsp. tremolsii). Furthermore, two other infraspecific taxa have been genetically confirmed as well-founded, i.e., E. helleborine subsp. distans (Arv.-Touv.) R.Engel and P.Quentin and E. helleborine subsp. voethii, although the latter one still has not been officially distinguished at this rank. As it appears, E. bugacensis Robatsch and E. rhodanensis Gévaudan and Robatsch have in fact a similarly close genetic relationship with E. dunensis (T.Stephenson and T.A.Stephenson) Godfery (originally included in the E. helleborine group), which, in our opinion, due to its floral morphologies (typical of autogamous taxa) should not be classified in this group. Although E. nordeniorum Robatsch was for a long time assigned to the E. helleborine group, as a result of recent genetic analysis it turned out to be molecularly similar to E. albensis Nováková and Rydlo, classified in a separate group. Some taxa, such as E. condensata Boiss. ex D.P.Young and E. cupaniana, based on results of a detailed morphological analysis of their floral and vegetative characters, should be retained in the E. helleborine group, although these taxa are still not included there in the most recently published accounts of the genus Epipactis.

The boundaries between individual species within the Epipactis helleborine group are unclear, making it difficult to determine reliable taxonomic characters useful in the construction of an identification key which would be unambiguously interpreted by different users. In the light of the scientific literature published worldwide, especially because of the impact of the genetic research on our current understanding of the boundaries between various species of Epipactis, we think it is appropriate to maintain the E. helleborine group, but we 
propose to update its circumscription to better reflect the taxonomic changes summarised in Table 1 that have occurred for its individual members over the past few decades.

Our proposed taxonomic circumscription of the Epipactis helleborine group therefore consists of the following only allogamous taxa: E. helleborine subsp. helleborine, E. helleborine subsp. bithynica, E. helleborine subsp. distans, E. helleborine subsp. neerlandica, E. helleborine var. tangutica, E. helleborine subsp. tremolsii, E. helleborine subsp. voethii (Robatsch) JakubskaBusse, Żołubak, and Łobas, stat. nov., E. condensata, E. condensata var. kuenkeleana and E. cupaniana.

Although the proposed list of taxa in the Epipactis helleborine group seems to be appropriate at the moment, we treat it as legitimate until new methods of genetic and morphometric analysis are developed, which would allow more precise definition of the Epipactis separate species concept in the future.

\section{Proposal of a New Status for Epipactis helleborine subsp. voethii}

Epipactis helleborine subsp. voethii (Robatsch) Jakubska-Busse, Żołubak, and Łobas, stat. nov.

Basionym: Epipactis voethii Robatsch, Mitteilungen der Abteilung für Botanik am Landesmuseum Joanneum in Graz 21/22: 22 (1993).

Comments: This subspecies differs from typical Epipactis helleborine s.str. through few developed clinandrium, as well as the slight differences observed in the morphological characters, i.e., the green colour of stems, leaves, and flowers of the individual plants, which are almost lacking in any violet coloration. In the fruiting stage, taxa can be distinguished by the shape of the seeds: in E. helleborine s.str. the seeds are worm-like, and club-shaped in E. helleborine subsp. voethii.

Author Contributions: Conceptualization, Z.Ł. and A.J.-B.; methodology, Z.Ł., E.Ż. and A.J.-B.; investigation, Z.Ł., E.Ż. and A.J.-B.; writing—original draft preparation, Z.Ł., A.K., E.Ż. and A.J.-B.; writing-review and editing, Z.E., A.K., E.Ż. and A.J.-B.; visualization, E.Ż. All authors have read and agreed to the published version of the manuscript.

Funding: This research received no external funding.

Institutional Review Board Statement: Not applicable.

Informed Consent Statement: Not applicable.

Data Availability Statement: Publicly available datasets were analysed in this study.

Acknowledgments: The authors wish to thank to Jacek Wajer (Natural History Museum) for improving the English version of the text.

Conflicts of Interest: The authors declare no conflict of interest.

\section{References}

1. Claessens, J.; Kleynen, J.; Wiellnga, R. Some Notes on Epipactis helleborine (L.) Crantz ssp. neerlandica (Vermeulen) Buttler and Epipactis renzii K. Robatsch. Eurorchis 1998, 10, 55-64.

2. Mered'a, P.; Potůček, O. Epipactis futakii, spec. nova (Orchidaceae)—Eine Neue Kleistogam Blühende Sitter—Art aus der Slovakei. Preslia 1998, 70, 247-258.

3. Chen, S.C.; Zhu, G. Nomenclatural changes in Epipactis (Orchidaceae) from China. Novon 2003, 13, 423-424. [CrossRef]

4. Hollingsworth, P.M.; Squirrell, J.; Hollingsworth, M.L.; Richards, A.J.; Bateman, R.M. Taxonomic complexity, conservation and recurrent origins of self-pollination in Epipactis (Orchidaceae). In Current Taxonomic Research on the British and European Flora; Bailey, J.P., Ellis, R.G., Eds.; Botanical Society of the British Isles: London, UK, 2006; pp. 27-44.

5. Efimov, P.G. Notes on Epipactis condensata, E. rechingeri and E. purpurata (Orchidaceae) in the Caucasus and Crimea. Willdenowia 2008, 38, 71-80. [CrossRef]

6. Szlachetko, D.L. Flora Polski. Storczyki; Multico: Warsaw, Poland, 2009.

7. Fateryga, A.V.; Kreutz, C.A.J. A new Epipactis species from the Crimea, South Ukraine (Orchidaceae). J. Eur. Orchid. 2012, 44, 199-206.

8. Fateryga, A.V.; Kreutz, C.A.J.; Fateryga, V.V.; Efimov, P.G. Epipactis krymmontana (Orchidaceae), a new species endemic to the Crimean Mountains and notes on the related taxa in the Crimea and bordering Russian Caucasus. Phytotaxa 2014, 172, $22-30$. [CrossRef] 
9. Chase, M.W.; Cameron, K.M.; Freudenstein, J.V.; Pridgeon, A.M.; Salazar, G.; Van den Berg, C.; Schuiteman, A. An updated classification of Orchidaceae. Bot. J. Linn. Soc. 2015, 177, 151-174. [CrossRef]

10. Fateryga, A.V.; Fateryga, V.V. The genus Epipactis Zinn (Orchidaceae) in the flora of Russia. Turczaninowia 2018, 21, 19-34.

11. Bateman, R.M. Implications of next-generation sequencing for the systematics and evolution of the terrestrial orchid genus Epipactis, with particular reference to the British Isles. Kew Bull. 2020, 75, 1-22. [CrossRef]

12. Clark, M. Epipactis leptochila var. cordata. J. Hardy Orchid Soc. 2021, 18, 26.

13. Squirrell, J.; Hollingsworth, P.M.; Bateman, R.M.; Tebbitt, M.C.; Hollingsworth, M.L. Taxonomic complexity and breeding system transitions: Conservation genetics of the Epipactis leptochila complex (Orchidaceae). Mol. Ecol. 2002, 11, 1957-1964. [CrossRef]

14. Krajnc, A.U.; Ivanuš, A.; Luthar, Z.; Lipovšek, M. Morphological variability and taxonomic concepts of Broad-leaved Helleborine ingroup Epipactis helleborine (L.) Crantz. Folia Biol. Geol. 2020, 61, 97-125. [CrossRef]

15. Ehlers, B.K.; Olesen, J.M.; Ågren, J. Floral morphology and reproductive success in the orchid Epipactis helleborine: Regional and local across-habitat variation. Plant Syst. Evol. 2002, 236, 19-32. [CrossRef]

16. Foley, M.; Clarke, S. Orchids of the British Isles; Griffin: Cheltenham, UK, 2005.

17. Delforge, P. Orchids of Europe, North Africa and the Middle East; A\&C Black Publishers Ltd.: London, UK, 2006.

18. Jakubska-Busse, A.; Gola, E.M. Morphological variability of Helleborines. I. Diagnostic significance of morphological features in Epipactis helleborine (L.) Crantz, Epipactis atrorubens (Hoffm.) Besser and their hybrid, Epipactis x schmalhausenii Richt. (Orchidaceae, Neottieae). Acta Soc. Bot. Pol. 2010, 79, 207-213. [CrossRef]

19. Jacquemyn, H.; van der Meer, S.; Brys, R. The impact of hybridization on long-term persistence of polyploid Dactylorhiza species. Am. J. Bot. 2016, 103, 1829-1837. [CrossRef]

20. Jakubska-Busse, A.; Żołubak, E.; Łobas, Z. Epipactis $\times$ subtilis (Orchidaceae), a new hybrid between E. albensis and E. purpurata. Ann. Bot. Fenn. 2017, 54, 139-144. [CrossRef]

21. Ivanuš, A. Morphological and Molecular Analyses of Individual Genotypes from the Group of Epipactis helleborine s.l. in the Region of Goričko. Diploma Thesis, University of Maribor, Maribor, Slovenia, 2018.

22. Jakubska-Busse, A.; Proćków, J.; Górniak, M.; Gola, E.M. Is Epipactis pseudopurpurata distinct from E. purpurata (Orchidaceae)? Evidence from morphology, anatomy, DNA and pollination biology. Bot. J. Linn. Soc. 2012, 170, 243-256. [CrossRef]

23. Brullo, C.; D'Emerico, S.; Pulvirenti, S. Karyological and taxonomical considerations on Epipactis cupaniana sp. nov. (Orchidaceae) from Sicily. Nord. J. Bot. 2013, 31, 577-589. [CrossRef]

24. Sramkó, G.; Paun, O.; Brandrud, M.K.; Laczkó, L.; Molnár, A.; Bateman, R.M. Iterative allogamy-autogamy transitions drive actual and incipient speciation during the ongoing evolutionary radiation within the orchid genus Epipactis (Orchidaceae). Ann. Bot. 2019, 124, 481-497. [CrossRef]

25. Sundermann, H. Europäische und Mediterrane Orchideen; Brücke Verlag Kurt Schmersow: Hildesheim, Germany, 1975.

26. Richards, A.J. The influence of minor structural changes in the flower on breeding systems and speciation in Epipactis Zinn. (Orchidaceae). In Pollination and Evolution; Armstrong, J.A., Powell, J.M., Richards, A.J., Eds.; Royal Botanic Gardens: Sydney, Australia, 1982; pp. 47-53.

27. Davies, P.; Davies, J.; Huxley, A. Wild orchids of Britain and Europe; The Hogarth Press: London, UK, 1983.

28. Buttler, K.P. Field Guide to Orchids of Britain and Europe; The Crowd Press: Marlborough, UK, 1991.

29. Delforge, P. Guide des Orchidées d'Europe d'Afrique du Nord et du Proche-Orient; Delachaux et Niestlé: Lausanne, Switzerland, 1994.

30. Delforge, P. Orchids of Britain and Europe. Collins Photo Guide; Harper Collins Publishers: London, UK, 1995.

31. Delforge, P. Orchidées d'Europe, d'Afrique du Nord et du Proche-Orient, 4th ed.; Delachaux et Niestlé: Paris, France, 2016.

32. Baumann, H.; Künkele, S.; Lorenz, R. Ulmer Naturführer Orchideen Europas mit Angrenzenden Gebieten; Ulmer Eugen Verlag: Stuttgart, Germany, 2006.

33. Xinqi, C.; Zhongjian, L.; Guanghua, Z.; Kaiyong, L.; Zhanhe, J.; Yibo, L.; Xiaohua, J.; Cribb, P.; Wood, J.; Gale, S.; et al. Flora of China, 25: Orchidaceae; Missouri Botanical Garden Press: Saint Louis, MO, USA, 2009.

34. Štěpánková, J.; Chrtek, J., Jr.; Kaplan, Z. Květena České republiky, 8th ed.; Academia: Praha, Czech Republic, 2011.

35. Mossberg, B.; Pedersen, H.A. Orchids; HarperCollins Publishers: Glasgow, Scotland, 2017.

36. Youssef, S.; Galalaey, A.; Mahmood, A.; Mahdi, H.; Véla, E. Wild Orchids of the Kurdistan Region Areas: A Scientific Window on the Unexpected Nature of the North-Western Zagros; Société Méditerrannéenne d'Orchidologie: La Motte-d'Aigues, France, 2019.

37. Cole, S.; Waller, M. Britain's Orchids: A Field Guide to the Orchids of Great Britain and Ireland; Princeton University Press: Woodstock, UK, 2020.

38. Efimov, P.G. Orchids of Russia: Annotated checklist and geographic distribution. Nat. Conserv. Res. 2020, 5 (Suppl. 1), 1-18. [CrossRef]

39. Rouhan, G.; Gaudeul, M. Plant taxonomy: A historical perspective, current challenges, and perspective. In Molecular Plant Taxonomy. Methods in Molecular Biology; Besse, P., Ed.; Humana: New York, NY, USA, 2021; Volume 2222.

40. Squirrell, J.; Hollingsworth, P.M.; Bateman, R.M.; Dickson, J.H.; Light, M.H.S.; MacConaill, M.; Tebbitt, M.C. Partitioning and diversity of nuclear and organelle markers in native and introduced populations of Epipactis helleborine (Orchidaceae). Am. J. Bot. 2001, 88, 1409-1418. [CrossRef]

41. Bateman, R.M. How many orchid species are currently native to the British Isles. In Current Taxonomic Research on the British and European Flora; Bailey, J.P., Ellis, R.G., Eds.; Botanical Society of the British Isles: London, UK, 2006; pp. 89-110. 
42. Tranchida-Lombardo, V.; Cafasso, D.; Cristaudo, A.; Cozzolino, S. Phylogeographic patterns, genetic affinities and morphological differentiation between Epipactis helleborine and related lineages in a Mediterranean glacial refugium. Ann. Bot. 2011, 107, 427-436. [CrossRef] [PubMed]

43. Holub, J. Epipactis leptochila (Godf.) Godf., a Epipactis muelleri Godf.-Nové druhy československé flóry. Preslia 1970, 42, 330-349.

44. Senghas, K.; Sundermann, H. Probleme der Orchideengattung Epipactis. Jahresberichte des Naturwissenschaftlischen Vereins in Wuppertal 1970, 23, 1-132.

45. Procházka, F.; Velísek, V. Orchideje Naší Př́rody; Academia Ved: Prague, Czech Republic, 1983.

46. Reinhard, H.R.; Gölz, P.; Peter, R.; Wildermuth, H. Die Orchideen de Schweiz und Angrenzender Gebiete; Fotorotar AG: Egg, Germany, 1991.

47. Tyteca, D.; Dufrêne, M. Biostatistical studies of western European allogamous populations of the Epipactis helleborine (L.) Crantz species group (Orchidaceae). Syst. Bot. 1994, 19, 424-442. [CrossRef]

48. Potůček, O.; Čačko, L'. V̌sechno o Orchidejích; Slovart: Prague, Czech Republic, 1996.

49. Szlachetko, D.L.; Skakuj, M. Storczyki Polski; Sorus: Poznań, Poland, 1996.

50. Mered'a, P. Kl'úč na určovanie druhov rodu Epipactis Zinn publikovaných z územia Slovenska. Bulletin Slovenskej Botanickej Spoločnosti. 1999, 21, 131-142.

51. Průša, D. Orchideje České Republiky; Computer Press: Brno, Czech Republic, 2005.

52. Průša, D. Orchideje České Republiky; CPress: Brno, Czech Republic, 2019.

53. Harrap, A.; Harrap, S. Orchids of Britain and Ireland. A Field and Site Guide; A\&C Black Publishers Ltd.: London, UK, 2009.

54. Batoušek, P.; Kežlínek, Z. Kruštiky České Republiky; Český svaz ochránců přírody ZO Hořepník: Prostějov, Czech Republic, 2012.

55. Bateman, R.M. Circumscribing species in the European orchid flora: Multiple datasets interpreted in the context of speciation mechanisms. Berichte aus den Arbeitskreisen Heimische Orchideen Beiheft 2012, 29, 160-212.

56. Ehlers, B.K.; Pedersen, H.Æ. Genetic variation in three species of Epipactis (Orchidaceae): Geographic scale and evolutionary inferences. Biol. J. Linn. Soc. 2000, 69, 411-430. [CrossRef]

57. Brzosko, E.; Wróblewska, A.; Talalaj, I. Genetic variation and genotypic diversity in Epipactis helleborine populations from NE Poland. Plant Syst. Evol. 2004, 248, 57-69. [CrossRef]

58. Bateman, R.M.; Hollingsworth, P.M.; Squirrell, J.; Hollingsworth, M.L. Tribe Neottieae. Phylogenetics. In Genera Orchidacearum 4. Epidendroideae 1; Pridgeon, A.M., Cribb, P.J., Chase, M.W., Rasmussen, F.N., Eds.; Oxford University Press: Oxford, UK, 2005.

59. Zhou, T.; Jin, X.H. Molecular systematics and the evolution of mycoheterotrophy of tribe Neottieae (Orchidaceae, Epidendroideae). PhytoKeys 2018, 94, 39-49. [CrossRef]

60. Xing, X.; Gao, Y.; Zhao, Z.; Waud, M.; Duffy, K.J.; Selosse, M.A.; Jakalski, M.; Liu, N.; Jacquemyn, H.; Guo, S. Similarity in mycorrhizal communities associating with two widespread terrestrial orchids decays with distance. J. Biogeogr. 2020, 47, 421-433. [CrossRef]

61. Lang, D. Britain's Orchids, a Guide to the Identification and Ecology of the Wild Orchids of Britain and Ireland; WILDGuides Ltd.: Old Basing, UK, 2004.

62. Wittig, R.; Wittig, M. Epipactis helleborine (L.) Crantz-The first (semi) ruderal orchid species of Central Europe. Feddes Repert. 2007, 118, 46-50. [CrossRef]

63. Kühn, R.; Pedersen, H.Æ.; Cribb, P. Field Guide to the Orchids of Europe and the Mediterranean; Kew Publishing: Kew, UK, 2019.

64. Kolanowska, M. Niche conservatism and the future potential range of Epipactis helleborine (Orchidaceae). PLoS ONE 2013, 8 , e77352. [CrossRef] [PubMed]

65. Rewicz, A.; Rewers, M.; Jędrzejczyk, I.; Rewicz, T.; Kołodziejek, J.; Jakubska-Busse, A. Morphology and genome size of Epipactis helleborine (L.) Crantz (Orchidaceae) growing in anthropogenic and natural habitats. PeerJ 2018, 6, e5992. [CrossRef] [PubMed]

66. Young, D.P. Bestimmung und Verbreitung der autogamen Epipactis-Arten. Jahresberichte Naturwissenschaftlichen Vereins Wuppertal 1970, 23, 43-52.

67. Bayer, M. Die Gattung Epipactis Zinn in Baden-Wuirttemberg. Mitteilungsblättern Arbeitskreis Heimische Orchideen BadenWürttemberg 1980, 12, 219-268.

68. Robatsch, K. Beitrage zur Bliitenbiologie und Autogamie der Gattung Epipactis. Jahresberichte Naturwissenschaftlichen Vereins Wuppertal 1983, 36, 25-32.

69. Engel, R. Epipactis allogames et autogames. Monde Plantes 1986, 425-426, 12-18.

70. Popovich, A.V.; Averyanova, E.A.; Shagarov, L.M. Orchids of the Black Sea coast of Krasnodarsky Krai (Russia): Current state, new records, conservation. Nat. Conserv. Res. 2020, 5 (Suppl. 1), 46-68. [CrossRef]

71. Kreutz, C.A.J. Kompendium der Europäischen Orchideen. Catalogue of European Orchids; Kreutz C.A.J.: Landgraaf, The Netherlands, 2004.

72. Delforge, P. Nouvelles contributions taxonomiques et nomenclaturales aux Orchidées d'Europe. Natural. Belges 2015, 96, 14-21.

73. POWO_Plants of the World Online. Facilitated by the Royal Botanic Gardens: Kew. 2019. Available Online: http://www. plantsoftheworldonline.org/ (accessed on 9 August 2021).

74. WCSP-World Checklist of Selected Plant Families. Facilitated by the Royal Botanic Gardens: Kew. 2021. Available Online: http:/ / wcsp.science.kew.org/ (accessed on 9 August 2021).

75. WCVP-World Checklist of Vascular Plants, version 2.0. Facilitated by the Royal Botanic Gardens: Kew. 2021. Available Online: http:/ / wcvp.science.kew.org/ (accessed on 9 August 2021).

76. WFO-World Flora Online. 2021. Available Online: http://www.worldfloraonline.org/ (accessed on 9 August 2021).

77. Linnaeus, C. Species Plantarum, 1st ed.; L. Salvius: Stockholm, Sweden, 1753. 
78. Govaerts, R.H.A. World Checklist of Monocotyledons Database in ACCESS: 1-71827; The Board of Trustees of the Royal Botanic Gardens: Kew, UK, 2003.

79. Govaerts, R.H.A. World Checklist of Selected Plant Families published update; Facilitated by the Trustees of the Royal Botanic Gardens: Kew, UK, 2011.

80. Govaerts, R.H.A. World Checklist of Vascular Plants (WCVP Database); The Board of Trustees of the Royal Botanic Gardens: Kew, UK, 2019.

81. Gruppo Italiano per la Ricerca sulle Orchidee Spontanee (G.I.R.O.S.). Orchidee d'Italia—Guida alle Orchidee Spontanee; Il Castello: Milano, Italy, 2009; Available Online: http:/ / www.giros.it/ (accessed on 9 August 2021).

82. Efimov, P.G.; Verkhozina, A.V. Epipactis helleborine var. tangutica (Orchidaceae), a new taxon for the flora of Russia and Middle Asia. Botanicheskii Zhurnal 2014, 99, 91-95.

83. Klein, E. Revision der spanischen Epipactis-Taxa E. atrorubens (Hoffm.) Schult. subsp. parviflora A. et C. Nieschalk, „E. atrorubentimicrophylla" und E. tremolsii C. Pau. Die Orchidee 1979, 30, 49-51.

84. Greuter, W.; Raus, T. Med-Checklist Notulae, 13. Willdenowia 1986, 16, 103-116. 